\title{
Expression and Immunogenicity Determination of Recombinant Clone of Pasteurella multocida Serotype B Against Haemorrhagic Septicaemia: Towards a Vaccine Development
}

\author{
${ }^{1} \mathrm{Jamal}$ Hussaini, ${ }^{2} \mathrm{Mahmood} \mathrm{A}$. Abdullah and ${ }^{2}$ Salmah Ismail \\ ${ }^{1}$ Faculty of Medicine, Universiti Teknologi MARA, 40450 Shah Alam, Selangor, Malaysia \\ ${ }^{2}$ Department of Molecular Medicine, Faculty of Medicine, University of Malaya, \\ 50603 Kuala Lumpur, Malaysia
}

\begin{abstract}
Clone ABA392 is virulent to mice and is capable of producing HS like symptoms. Balb/C mice were injected with various forms of vaccine (live and killed) and vaccination schedules in order to determine the immunogenicity of the clone. The immunized animals were challenged with lethal dose of Pasteurella multocida serotype B and at the same time a Passive Mice Protection Test (PMPT) was also carried out. Protection of 66 and $83 \%$ was achieved in mice vaccinated with live and killed forms, respectively. In PMPT, $66 \%$ of mice passively immunized with immune sera were protected. By performing ELISA test, it was found that the triple dose vaccination elicited high antibody production in the vaccinated animals. There was significant increase $(\mathrm{p}<0.05)$ antibody production in triple dose vaccination compared to double dose vaccination. The clone ABA392 was sub-cloned into an expression system. The sub-clone (CSI57J) was expressed and with the help of immuno-detection method (chromogenic method) specific to His-tagged expressed protein, the expression was confirmed. The expressed protein was extracted, purified and quantified and subsequently subjected to SDS-PAGE for size detection. The protein size was found to be about $26 \mathrm{kDa}$. Mice were immunized with the expressed protein and the effect of direct and passive immunization was determined. In direct immunization, $90 \%$ of mice were protected from lethal dose of $P$. multocida serotype $\mathrm{B}$ and on the other hand, $83 \%$ protection was observed in passively vaccinated mice. This study shows that the expressed sub-clone CSI57J has the potential to be a good candidate for a new recombinant vaccine against HS.
\end{abstract}

Key words: Immunogenicity, recombinant clone, Pasteurella multocida serotype B, vaccine, candidate, protection, mice

\section{INTRODUCTION}

Haemorrhagic Septicaemia (HS) is considered as a major and one of the most important infectious diseases of cattle and buffalo. In some countries, particularly Eastern Asian countries, animals play an important integrated role in rural communities as a main source of milk, fuel, meat and fertiliser. The HS epidemics can have disastrous consequences; not only has the loss of live-stocks but also affect economical returns, resulting in reduced productivity and livelihood of villagers and farmers (Benkirane and De Alwis, 2002). HS due to P. multocida serotype B causes loss of millions of dollars to the farming industry worldwide annually. In order to control and minimise the losses, wide range of preventive measures, predominantly in vaccine development against HS has been carried out (Dabo et al., 2008). There have been several types of vaccines developed with very limited protection. At the time when this study was carried out, no recombinant single antigen had been identified to stimulate protection against $P$. multocida serotype B. The clone ABA392 used in this study is derived from a P. multocida serotype $\mathrm{B} 202$, carrying a recombinant plasmid. This clone was constructed by shotgun cloning method using $E$. coli as a host. The recombinant plasmid previously sequenced (Salmah, 2000) harbours a sequence that code for a virulence factor to $P$. multocida. The insert was found to be $921 \mathrm{bp}$. The aim of this study was to determine the immunogenicity of the clone, sub-cloning into an expression system and its potency as a possible vaccine candidate.

\section{MATERIALS AND METHODS}

Bacterial isolates: The Pasteurella multocida isolates used in this study were provided by Dr. Salmah Ismail,

Corresponding Author: Jamal Hussaini, Faculty of Medicine, Universiti Teknologi MARA, 40450 Shah Alam, Selangor, Malaysia 
Faculty of Medicine, University of Malaya, Malaysia. The recombinant clone ABA392 previously produced (Salmah, 1997) was used in this study.

Animal experiments: About 4-6 weeks old pathogen free $\mathrm{Balb} / \mathrm{C}$ mice weighing between 18-25 g were used for this part of experiment. Mice were obtained from the Animal House, Faculty of Medicine, University of Malaya. The animals were housed in cages at the experimental rooms, Animal House, Faculty of Medicine, University of Malaya and were fed a commercial feed along with water. Preparation of bacterial suspension for vaccine was adapted from Mariana and Hirst in 2000 with modification. A single pure bacterial culture was grown overnight at $37^{\circ} \mathrm{C}$ in $10 \mathrm{~mL}$ of $\mathrm{BHI}$ broth medium. The cultures were then centrifuged for $5 \mathrm{~min}$ at $14000 \mathrm{rpm}$ to pellet the cells. The pelleted cells were washed three times with sterile PBS to remove all traces of the growth medium. The cells were resuspended in $5 \mathrm{~mL}$ of PBS pH 7.2 to achieve the concentration of $10^{7}$ colony forming units (cfu).

Formalin of $0.2 \%$ concentration was added. The cell suspensions were left to stand for $24 \mathrm{~h}$ and then centrifuged at $14000 \mathrm{rpm}$ for $5 \mathrm{~min}$ and the supernatant was replaced by an equal volume of fresh $0.2 \%$ formalinized PBS. Mice were divided into different groups where each group was injected intraperitoneally with $1 \mathrm{~mL}$ $\left(10^{7} \mathrm{cfu}\right)$ of either killed or live strains of clone ABA392 or P. multocida serotype B202. The bacterial strains were prepared in Freund's complete adjuvant. The vaccination was carried out either with a double dose or triple dose schedules. In double dose schedule, the vaccines were given on day zero followed by a booster dose 2 weeks later. Whereas the three dose schedule, vaccination was on day 0 , weeks 2 and 4 intervals. Animals were inspected twice daily and their conditions were monitored. Any changes observed in behaviour, movement, eating and development of any signs and symptoms were recorded. Serum was collected from all the mice before vaccination and 1 month post vaccination. At the end of the vaccination, all mice with various vaccination schedules were challenged by i.p. injection of $10^{9} \mathrm{cfu} P$. multocida serotype B202 parental strains 1 month after primary vaccination.

Passive mice protection test: Pooled sera sample was prepared from each group of immunized rats and $0.5 \mathrm{~mL}$ of it was then injected into $6-8$ weeks old $\mathrm{Balb} / \mathrm{C}$ mice. After $24 \mathrm{~h}$ the mice were then challenged by i.p. injection of lethal doses of $10^{9}$ cfu P. multocida serotype B202 and were kept under observation for 10 days. The percentage of mice surviving and the time of death were recorded. The percentage of mice surviving and the time of death were recorded. At the end of the vaccination mice were sacrificed by cervical dislocation following the animal ethics requirements. Blood was collected and serum was separated, pooled and stored at $-20^{\circ} \mathrm{C}$ until it was used. Total white blood cells and differential count was performed using automated blood analyzer. The organs of sacrificed mice were collected at the end of the experiment for gross and histopathological analysis. Autopsy was performed on the mice after they were euthanized by being placed in jar containing diethyl ether. Lungs, liver and kidneys were collected and fixed in $10 \%$ formalin and then trimmed before they were processed. The tissue samples were processed in an automated tissue processor Leica TP1020 for about $15 \mathrm{~h}$. After the processing procedure the tissues were embedded into paraffin blocks using tissue embedding machine (Histo embedder leica) for sectioning of the tissues with leica RM2035 microtome the tissues were sectioned to $15 \mu \mathrm{m}$ thickness using a sharp unused microtome blade for best result. The cut sections of tissues were gently placed on a warm water bath $\left(40^{\circ} \mathrm{C}\right)$ to remove the wrinkles. The tissue sections then picked up with glass slides, the slides then placed on a slide warmer with $55^{\circ} \mathrm{C}$ temperature for about $15 \mathrm{~min}$ or till the slides are dried. This process will help the sections to adhere to the slides. The staining of the slides involves several steps, commencing with removal of the paraffin wax. The slides were stained with Harris haematoxylin stain for $10 \mathrm{~min}$.

Enzyme Linked Immuno-Sorbent Assay (ELISA): ELISA was developed based on the procedure by Pati et al. (1996) with modifications. ELISA was performed on the hyper-immune sera obtained from the immunized mice for presence of antibody. About $100 \mu \mathrm{L}$ of antigen was coated on the 96 well microtiter plate, sealed and kept overnight at $4^{\circ} \mathrm{C}, 100 \mu \mathrm{L}$ of serum was then added and incubated for $1 \mathrm{~h}$ at room temperature followed by 3 times wash with phosphate buffered saline with tween 20 (PBS-Tween 20) by using a multi-channel pipette. About $100 \mu \mathrm{L}$ of diluted Anti-mouse IgG $(\mathrm{H}+\mathrm{L})$ Alkaline Phosphatase conjugate was then added followed by washing step as mentioned. About $200 \mu \mathrm{L}$ of the substrate was added and incubated for $30 \mathrm{~min}$ at room temperature and finally the reaction was stopped by adding $200 \mu \mathrm{L} \mathrm{NaOH}$. The absorbance $\mathrm{OD}$ was read at 405 nm. All samples, blanks, positive and negative controls were used in triplicates. The positive control samples were obtained from mice which had been hyper-immunised with $P$. multocida serotype B. The cells were grown in $500 \mathrm{~mL}$ of $\mathrm{BHI}$ broth at $37^{\circ} \mathrm{C}$. The harvested culture were washed in PBS twice and then resuspended in fresh $0.2 \%$ formalinized PBS. This process inactivates 
the organism after $24 \mathrm{~h}$ of incubation. The density of the suspension was adjusted till a concentration of $10^{7} \mathrm{cfu}$ was achieved. The negative control were serum collected from healthy mice not exposed to $P$. multocida and injected with PBS as mentioned above. Cut-off value was determined using the Two-graph Receiver Operating Characteristic (TG-ROC) a Microsoft Excel Program (Greiner, 1995; Greiner et al., 1995). The ELISA results were analysed based on the cut-off value including sensitivity and specificity obtained from TG-ROC.

Sub-cloning of the ABA392 into an expression system: Sub-cloning of the insert ABA 392 was carried out using $\mathrm{pQE}$ expression system as described in QIA expressionist with modification. The $\mathrm{pQE}$ expression construct was created by ligation and transformation into the M15 host strain carrying the pREP4 repressor plasmid. Transformants were then selected on plates containing both ampicillin and kanamycin (Sambrook et al., 1989). The steps involved in producing the recombinant protein are cloning into an expression plasmid, selecting and growing of the cells with expressed plasmid, induction and purification as described in QIAexpressionist with modification. Immuno-detection was carried out to confirm that the sub-clone CSI57J has expressed 6xHis-tagged protein or not. This stage was carried out in two steps, first was colony blot in which the expressed colonies were transformed on to a nitrocellulose membrane and second was Immuno-detection with Anti-His antibodies Conjugates.

Protein separation technique SDS-PAGE: Protein separation was conducted via Sodium Dodecyl Sulfate Polyacrylamide Gel Electrophoresis (SDS-PAGE) technique. The method adopted in this study is taken from Laemmli, 1970. In this study, one set of SDS-PAGE was run using dual vertical mini gel unit (C.B.S. Scientific Inc., USA) with the gel size of $10 \times 10 \mathrm{~cm}$.

Statistical analysis: SPSS Version-12 and Microsoft Excel were used as statistical tool in processing and analyzing all data in this study.

\section{RESULTS AND DISCUSSION}

Mice challenge: Mice vaccinated with 2 dose of live ABA392 were not protected with the vaccine and all the mice in the group died after being challenged with PMB202. The 3 doses vaccination provided $66 \%$ protection whereas the mice vaccinated with 2 and 3 doses of live P. multocida serotype B202 16 and 66\% of them survived the challenge, respectively. All the mice died in the group which was vaccinated with 2 doses of killed ABA392. On the other hand, $83 \%$ of the mice survived in the group which received 3 doses of killed ABA392. The group vaccinated with 2 and 3 doses of killed P. multocida serotype B202 showed 66 and 100\% survival rate. In the non-vaccinated control group all the mice died (Table 1). The results of PMPT are shown in Table 2 .

Sub-cloning into expression vector: The clone was successfully sub-cloned into an expression vector $\mathrm{pQE} 32$ expression system and the new clone was named CSI57J for reference based on the clone number which showed positive for carrying the insert ABA392. The clone size was found to be $4.4 \mathrm{~kb}$ (Fig. 1-4) after the agarsoe gel

Table 1: No. of mice with various vaccinations surviving the challenge with PMB202

Bacterial strain, type and

vaccination interval

ABA392 Live OAV 2 doses

ABA392 Live OAV 3 doses

PMB202 Live OAV 2 doses

PMB202 Live OAV 3 doses

ABA392 killed OAV 2 doses

ABA392 killed OAV 3 doses

PMB202 killed OAV 2 doses

PMB202 killed OAV 3 doses

Not vaccinated No. of mice survived ${ }^{*} \quad$ Survived (\%)

$0 / 6 \quad 0.0$

$4 / 6 \quad 66.7$

$1 / 6 \quad 16.7$

$\begin{array}{ll}4 / 6 & 66.7\end{array}$

$0 / 6$

$5 / 6$

$4 / 6$

$6 / 6$

$0 / 6$

0.0

83.3

66.7

100.0 0.0

Table 2: Passive mice protection test result. Survival rate of mice after receiving different serum samples from various vaccination schedules and challenged with PMB202

\begin{tabular}{lcc}
\hline Mice and various immune sera & PMPT $^{*}$ & Survived (\%) \\
\hline ABA392 Live OAV 2 doses & $0 / 6$ & 0.0 \\
ABA392 Live OAV 3 doses & $4 / 6$ & 66.7 \\
PMB202 Live OAV 2 doses & $4 / 6$ & 66.7 \\
PMB202 Live OAV 3 doses & $4 / 6$ & 66.7 \\
ABA392 killed OAV 2 doses & $1 / 6$ & 16.7 \\
ABA392 killed OAV 3 doses & $4 / 6$ & 66.7 \\
PMB202 killed OAV 2 doses & $4 / 6$ & 66.7 \\
PMB202 killed OAV 3 doses & $6 / 6$ & 100.0 \\
Not vaccinated & $0 / 6$ & 0.0 \\
\hline
\end{tabular}

*No. of mice surviving/total no. of challenged

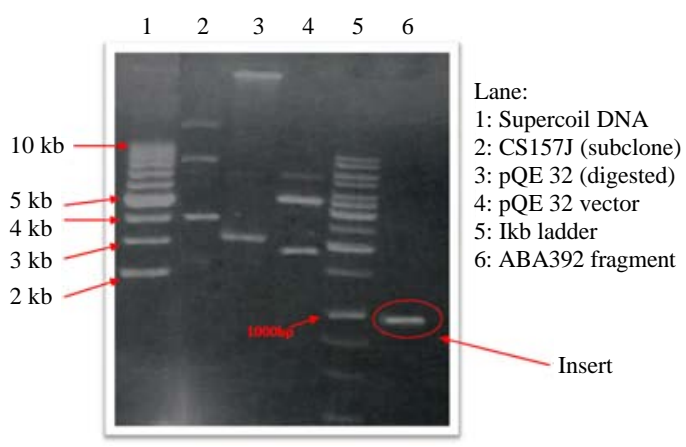

Fig. 1: Electrophoresis gel showing the clone CSI57J, vector $\mathrm{pQE}$ and the insert $\mathrm{ABA} 392$ 


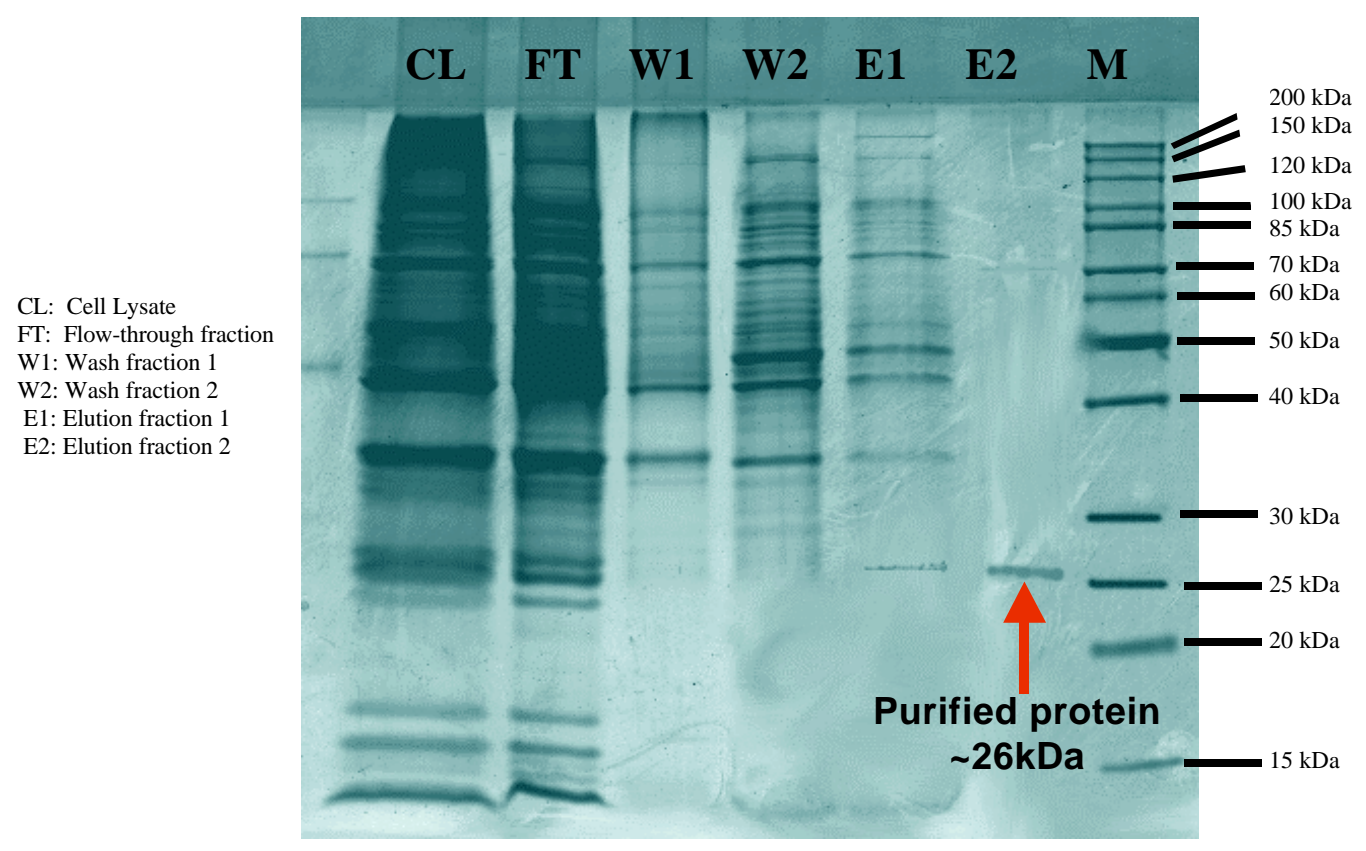

Fig. 2: SDS-PAGE gel showing 6xHis-tagged purified protein (red arrow) under native condition. SDS-PAGE result shows the approximate size of the recombinant protein determined to be $\sim 26 \mathrm{kDa}$

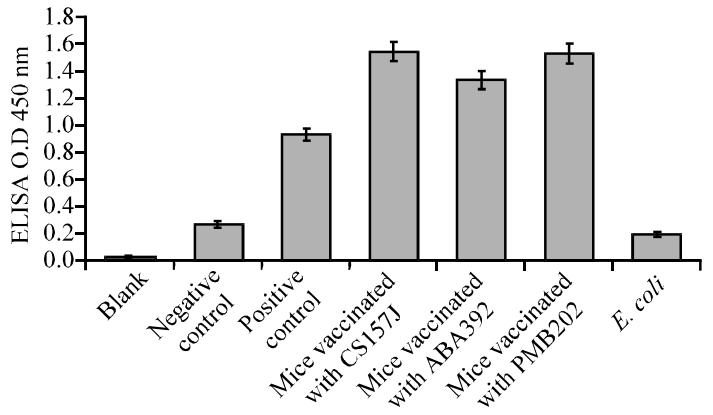

Types of vaccination
Fig. 3: ELISA O.D reading using $540 \mathrm{~nm}$ filter, performed on serum from mice vaccinated with CSI57J purified protein and compared with that of mice vaccinated with $\mathrm{ABA} 392$ and $\mathrm{PMB} 202$

electrophoresis analysis using supercoil ladder and $\mathrm{pQE}$ vector as controls and size identification (Fig. 1-4). Usually, local isolates are used in vaccine development. In the earliest form of HS vaccine, bacterins were used; furthermore alum or aluminum hydroxide gel vaccines were introduced (Chandrasekaran et al., 1994a, b). Oil adjuvant with killed whole cell were used in control HS (Opacka and Kedrak, 2003). To achieve better immunogenic vaccine, fractionation of $P$. multocida has been carried out and various cell components have been studied (Kennett et al., 1993; Srivastava, 1998; Confer et al., 2001).

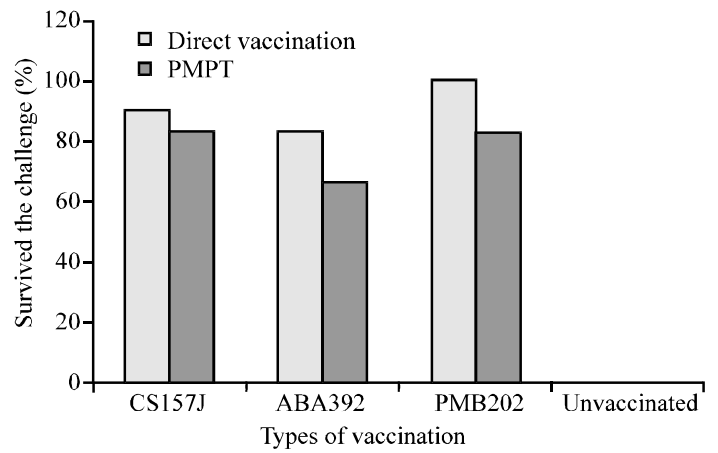

Fig. 4: Comparison between the immunity conferred by the ABA392 and the expressed protein as vaccines

An effective treatment for HS is difficult not only due to sudden onset of symptoms and death of animals but also due to the rising number of antibiotic resistance strains (Bindu et al., 2008). Hence, vaccination would be a better and cheaper option in HS control. Live attenuated vaccines have proven to be more effective, immunogenic and provide better protection than some of the killed preparations because of natural route of entry and can stimulate similar site as of the natural infection. This will only be effective if the modes of attenuation are well defined (Tabatabaei et al., 2007a, b). However, the role of cell mediated immune responses and antibody reactions are poorly understood (Verma and Jaiswal, 1998). ABA392 when used as killed or live attenuated vaccine in 2 dose 
2 weeks apart, provided no protection to the vaccinated mice and serum of the mice showed a negative result based on the cut-off valve obtained by TG-ROC analysis (Fig. 3). The 3 dose vaccination provided $66 \%$ protection and ELISA results showed a high titer of antibody production. The serum from these mice used in PMPT study provided $66 \%$ protection as well.

At the same time the similar vaccination regime was carried with $P$. multocida serotype $\mathrm{B}: 2$, the parental strain. The two dose vaccination was shown to be ineffective and the ELISA revealed a low titre of antibody while the 3 dose vaccination had provided almost a 100\% protection to mice with a very high antibody titre. In order to find the potency of the gene, the insert from the clone ABA392 was sub-cloned in to an expression system, expressed and purified and used for immunogenicity study. The new expressed clone (CSI57J) when injected into mice resulted in death of mice within $36 \mathrm{~h}$ of injection and upon histopatological study all sign and symptoms of HS were seen. The protein was extracted, purified and confirmed by immunodetection (Chromogenic Method). From SDS-PAGE the size of the protein was estimated to be $26 \mathrm{kDa}$. Information on antigens of $P$. multocida that are capable of stimulating immune response in cattle is limited. Some of the known effective immunogens of $P$. multocida serotype B causing HS are capsular antigen, LPS and outer membrane proteins (Opacka and Kedrak, 2003; Adlam, 1989).

The immunogenicity of CSI57J protein was determined and $90 \%$ protection was achieved with 3 dose vaccination and a high titre antibody was obtained on ELISA based on the cut-off value obtained from TG-ROC and $83 \%$ of mice survived the challenge with virulent strain of $P$. multocida serotype B:2 when they were passively immunized with serum from vaccinated mice prior to challenge. Upon vaccination mice showed no significant signs or reaction or lesions at the site of injection. Even though, the protection provided by this protein was lower than the whole cell vaccine, it is still above the acceptable level which is $67 \%$. Serum from mice immunized with the protein (CSI57J) showed positive radial immunodiffusion test, signifying that the sera of immunized mice comprised of antibody specific to the protein while the sera of non-immunized mice did not show any reaction towards the protein in this test.

\section{CONCLUSION}

This study was focused on the immunological response and introducing a potential candidate (ABA392 and expressed CSI57J) for vaccine production against haemorehagic septicaemia. The clone had proven to cause
HS in experimental animals (mice and rats) and most importantly to elicit antibody production when used as live or killed vaccines and protect mice from challenge with virulent strain of $P$. multocida serotype B:2. This study has proven that immune sera from vaccinated animals when administered intramuscularly to mice could protect them from virulent effects of $P$. multocida serotype B:2. No anaphylactic responses (shock) or any other reactions were observed in vaccinated animals. At the site of injection no inflammation or any other changes were detected.

This finding signifies that CSI57J as vaccine does not cause any visible detectable changes to the host. The capability of the purified expressed protein as an immunogen and eliciting high titer production of antibody against HS are evident in this study and researchers believe it can be a good vaccine candidate against HS. In order to discover the full potential of the clone, safety and efficacy of the protein in cattle will be focus of future study.

\section{REFERENCES}

Adlam, C., 1989. Haemorrhagic Septicaemia. In: Pasteurella and Pasteurellosis, Adlam, C. and J.M. Rutter (Eds.). Academic Press, London, UK., ISBN: 0120442744 , pp: $131-160$.

Benkirane, A. and M.C.L. De Alwis, 2002. Haemorrhagic septicaemia, its significance, prevention and ontrol in Asia. Rev. Art. Vet. Med. Czech, 47: 234-240.

Bindu, N., L.M. Lawrence, S.P. Divya and C.B. Shane, 2008. Effects of subminimum inhibitory concentrations of antibiotics on the Pasteurella multocida proteome: In a systems approach. Comp. Funct. Genomics, 10.1155/2008/254836.

Chandrasekaran, S., L. Kennett, P.C. Yeap, N. Muniandy, B. Rani and T.K. Mukkur, 1994a. Characterization of immune response of protection in buffaloes immunized with haemorrhagic septicaemia vaccines. Vet. Microbiol., 41: 213-219.

Chandrasekaran, S., L. Kennett, P.C. Yeap, N. Muniandy, B. Rani and T.K. Mukkur, 1994b. Relationship between active protection in vaccinated buffaloes against haemorrhagic septicaemia and passive mouse protection test or serum antibody titres. Vet. Microbiol., 41: 303-309.

Confer, A.W., M.A. Suckow, M. Montelongo, S.M. Dabo, L.J. Miloscio, A.J. Gillespie and G.L. Meredith, 2001. Intranasal vaccination of rabbits with Pasteurella multocida, A: 3 outer membranes that express iron-regulated proteins. Am. J. Vet. Res., 62: $697-703$ 
Dabo, S.M., A. Confer, M. Montelongo, P. York and J.H. Wyckoff, 2008. Vaccination with Pasteurella multocida recombinant Ompa induces strong but non-protective and deleterious Th2-type immune response in mice. Vaccine, 26: 4345-4351.

Greiner, M., 1995. Two-graph receiver operating characteristics (TG-ROC): A microsoft-excel template for the selection of cut-off values in diagnostic tests. J. Immunol. Meth., 185: 145-146.

Greiner, M., D. Sohr and P. Gobel, 1995. A modified ROC analysis for the selection of cut-off values and the definition of intermediate results of serodiagnostic tests. J. Immunol. Meth., 185: 123-132.

Kennett, L., N. Muniandy and T.K. Mukkur, 1993. Comparative Protection Potential of Non-Living Intact Cells and Purified Outer Membranes and Associated Proteins of Pasteurella Multocida Type 6: B Grown Under Iron-Regulated Conditions. In: Pasteurellosis in Production Animals, Patten, B.E., T.L. Spencer, R.B. Johnson, D. Hoffmann and L. Lehane (Eds.). Australian Centre for International Agricultural Research (ACIAR), Brisbane, Australia, pp: 44-148.

Opacka, B.B. and A. Kedrak, 2003. Evaluation of immunogenicity of outer membrane proteins of Pasteurella multocida serotype B:2,5 in cattle Bull. Vet. Res. Inst. Pulawy, 47: 377-385.

Pati, U.S., S.K. Srivastava, S.C. Roy and T. More, 1996. Immunogenicity of outer membrane protein of Pasteurella multocida in buffalo calves. Vet. Microbiol., 52: 301-311.
Salmah, I., 1997. Molecular studies Of Pastuerella multocida animal isolates. M.Sc. Thesis, University of Malaya, Kuala Lumpur, Malaysia.

Salmah, I., 2000. Molecular characterization of virulence-determinant of recombinant clone ABA392 from Pasteurella multocida PMB202 of serotype B animal isolate. $\mathrm{PhD}$. Thesis, University of Malaya, Kuala Lumpur, Malaysia.

Sambrook, J., E.F. Fritsch and T.A. Maniatis, 1989. Molecular Cloning: A Laboratory Manual. 2nd Edn., Cold Spring Harbor Laboratory Press, New York, USA., ISBN-13: 9780879695774, Pages: 3.

Srivastava, S.K., 1998. Outer membrane protein of Pasteurella multocida serotype B: 2 is immunogenic and antiphagocytic. Ind. J. Exp. Biol., 36: 530-532.

Tabatabaei, M., J.G.R. Moazzeni, Jabbari, A.R. and M. Esmailzadeh, 2007a. Pathogenicity and immunogenicity of native and mutant strains of Pasteurella multocida the causative agents of haemorrhagic septicaemia. Iran. J. Vet. Res., 8: 1-18.

Tabatabaei, M., G.R.J. Moazzeni, A.R. Jabbari and M. Esmailzadeh, 2007b. Vaccine efficacy in cattle against hemorrhagic septicemia with live attenuated aroA mutant of Pasteurella multocida B: 2 strain. J. Cell Anim. Biol., 1: 062-065.

Verma, R. and T.N. Jaiswal, 1998. Haemorrhagic septicaemia vaccines. Vaccine, 16: 1184-1192. 